American Journal of Pharmaceutical Education 2016; 80 (8) Article 135.

\title{
RESEARCH
}

\section{Evidence of Criterion Validity for One Pharmacy School's Progress Examination Program}

\author{
Greg L. Alston, PharmD, Wes R. Haltom, PharmD \\ Wingate University School of Pharmacy, Wingate, North Carolina \\ Submitted August 11, 2015; accepted February 7, 2016; published October 25, 2016.
}

Objective. To provide evidence that the progress examination program accurately assesses student failure to demonstrate competence.

Methods. A progress examination program aligned with each grade level was locally developed and administered annually to 289 PharmD students in the spring of their first year - fourth year. Correlations and linear regressions were performed to compare the examination scores to performance on national licensing examinations and cumulative didactic grade point average (GPA). Odds ratio analysis was run to determine the ability of the passing scores of the progress examination to identify students at increased risk of failing to graduate on time, earn a GPA below 3.0, and fail the licensing examinations on their first attempt.

Results. Progress examination scores were strongly correlated to GPA and national licensing examination scores and weakly correlated to jurisprudence examination scores. Regression analysis indicated a significant linear relationship between examination scores and both GPA and the licensing examinations. Students who performed poorly on the progress examinations were more likely fail the national licensing examination, more likely to fail to graduate on time, and more likely to earn a cumulative didactic GPA below 3.0.

Conclusions. The second-year examination program strongly predicts students at risk for failure to graduate on time or achieve a GPA below 3.0, while all four examinations identify students at risk of failing the national licensing examination on their first attempt.

Keywords: assessment, educational outcomes, progress examination, milemarker examination, student progression, PCOA

\section{INTRODUCTION}

Many schools use some form of progress examination to assess student performance. Vyas et al identified 52 pharmacy schools in the United States with some form of a cumulative progress examination. ${ }^{1}$ One major goal of these programs is to comply with accreditation standards, such as Standard 24 of the Accreditation Council for Pharmacy Education (ACPE) Standards 2016, which seeks to determine whether students are ready to pursue their final year of advanced practice experiences. ${ }^{2}$ Ultimately, justifying valid conclusions and decisions for the progress of individual doctor of pharmacy (PharmD) students requires evidence of validity for use of an assessment such as a progress examination. In the past, validity was defined as three separate types: content, criterion, and construct, with concurrent and predictive validity based on the timing of data collection. ${ }^{3}$

Corresponding Author: Greg L. Alston, Campus Box 3087, Wingate University School of Pharmacy, Wingate, NC 28174-0159. Tel: 704-233-8329. Fax: 888-870-2519. E-mail: greg@greglalston.com
Downing described validity as a unitary concept with construct validity as the whole of validity. ${ }^{3}$ In this view, validity is approached as a hypothesis. Assessments such as progress examinations are neither valid nor invalid. Assessment scores simply have more or less validity evidence to support their use for the proposed interpretation. Downing described five sources of validity evidence: (1) content; (2) response process; (3) internal structure; (4) relationship to other variables; and (5) consequences. While Downing's article reviewed validity as described in the 1999 Standards for Educational and Psychological Testing (SEPT), the SEPT 2014 retains this position when it states that "validity refers to the degree to which evidence and theory support the interpretations of test scores for proposed uses of tests." 4

The Annual Skills Mastery Assessment (ASMA) examination program was developed by faculty members at Wingate University School of Pharmacy (WUSOP) as a criterion-referenced annual progress examination. The ASMA examination compares students to a faculty panel's 


\section{American Journal of Pharmaceutical Education 2016; 80 (8) Article 135.}

estimate of competency on the grade-level appropriate skills [first year (P1) - fourth year (P4)] assessed on the examination. Evidence of validity should include how well the examination includes important content, aligns content with the curriculum, reflects what should be learned, reflects individual student scores in a meaningful way, allows cost-effective production, and delivers results in a timely fashion while measuring what it purports to measure. ${ }^{5}$ Previous articles explored the content, response process, and internal structure validity of the ASMA examinations, as well as the cut score development process. ${ }^{6,7}$ This study was designed to complete the initial validation process by analyzing the relationship to other variables and determining whether the cut scores identify students with increased likelihood of failure and thus can be predictive of competence (consequences).

\section{METHODS}

The development of the examination was described in detail in $2010 .^{6}$ Briefly, faculty members developed a list of Terminal Ability- Based Outcomes statements (TABO) in 2003. These statements were categorized as abilities that should be developed during the first, second, third, or fourth year of the curriculum. Test items were written by faculty members and designed to assess practice skills rather than factual knowledge because knowledgebased examinations can potentially encourage memorization over higher levels of learning. ${ }^{4}$

Faculty panels then used an Angoff scoring process to determine the minimal level of performance (cut score) on each test item. The Angoff process produces a reliable cut score for this examination. ${ }^{7}$ The cut scores for all test items are averaged to create a cut score for each subscale and for the entire examination. Each student receives a customized individual score report that summarizes progress in mastering the TABO-specific subscores as well as the aggregate ASMA examination score. The score reports are distributed via the student's faculty advisor to facilitate a frank end-of-grade formative progress discussion with each student (Appendix 1).

Student performance data is aggregated to produce TABO-specific summative evidence of curricular learning outcomes for the curriculum committee called the Mastery Summary Report (Appendix 2). The multiplechoice, 4-option, single best answer examination has been used since 2008 to track the ability of the WUSOP curriculum to develop targeted program outcomes in students as they progress through the curriculum. In addition, student aggregate data can be studied to examine intercampus performance as well as current success vs historical trends. The outcomes assessment committee selects the TABO to be tested each year and develops the test blueprint (Appendix 3). The students are not advised about examination content so they cannot skew the results by selectively studying for the examinations. All classes and practice experiences are cancelled and all tests are administered to all takers on a single day in late March each year. This assessment is designed to measure retention rather than short-term recall.

Reliability is the ability of an instrument to measure consistently. ${ }^{8}$ Internal consistency reliability is the test's correlation with itself. ${ }^{8}$ The ASMA examination metrics include Cronbach alpha, a measure of internal reliability, and the standard error of measurement (SEM), which is an estimation of measurement error. ${ }^{8,9}$ Cronbach Alpha over a 4-year period for P1 through P4 examinations ranged from a low of 0.69 to 0.88 . The SEM describes the effect of measurement error on the observed score of an individual student. ${ }^{9}$ It estimates how repeated measures of a student on the same test tend to be distributed around his or her "true" score. The SEM ranged from 3.7 to 4.8, which equates to a measurement error between 2.4-3.7 points per 100 point examination. Roughly $68 \%$ of student scores would fall within 1 SEM on repeated retests and $95 \%$ within 2 SEM.

The project described herein was approved by the Wingate University research review board. ChisholmBurns et al suggested that grade point average (GPA), North American Pharmacist Licensure Exam (NAPLEX) scores, and Multistate Pharmacy Jurisprudence Exam (MPJE) scores could be used as proxies for student success. ${ }^{10}$ These authors selected failure to achieve a GPA above 3.0 at the end of the didactic portion of the curriculum, failure to pass the NAPLEX on the first attempt, failure to pass MPJE on the first attempt, and failure to graduate on time (GOT) with their entering student cohort as proxies for failure to demonstrate competency in the abilities necessary to enter practice. Semester 6 GPA at the end of the didactic curriculum was used based on concerns that practice experience grades tend to be inflated, with at least one school reporting that $90 \%$ of them were As. ${ }^{11}$

For the analysis in this study, student performance data for the graduating classes of 2011 to $2014(\mathrm{~N}=290)$ were collected. Students who withdrew from the program prior to receiving any official grades for the first semester were excluded $(n=1)$. Data collected included the percentage correct ASMA examination score for the P1, $\mathrm{P} 2, \mathrm{P} 3$ and $\mathrm{P} 4$ examinations, the cumulative GPA at the end of the didactic curriculum (GPA6), and whether or not the student graduated from the program on time with their entering cohort. Data collected that was external to the WUSOP program included the examination score for the NAPLEX and MPJE examinations. Some students may 


\section{American Journal of Pharmaceutical Education 2016; 80 (8) Article 135.}

take an ASMA examination more than once before they proceed to the next didactic year because they have academically repeated a year. The ASMA examination score reported in these data was for the first attempt of the test for that didactic year only.

Additional analysis fields were computed from the original data points. Each ASMA examination score was analyzed to determine if the student score: (1) fell below the mastery cut score for the examination; (2) fell within 1 SEM of the cut score; or (3) fell within 2 SEM of the cut score. New study variables were created to account for each of these three cases. This analysis was added to explore the chance that measurement error internal to the ASMA examinations may fail to identify a low performer if WUSOP relied solely on the cut score rather than including students who barely exceeded the cut score. In addition, each ASMA score was converted to a z-score using EzAnalyze Software (Boston, MA). The data was then dichotomized in to five groups based on z-score. The groups were: (1) below 2 standard deviations below the mean; (2) between -1 and -1.99 standard deviations below the mean; ( 3 ) between -0.99 and +1 standard deviations above the mean, 4) greater than 1 but less than 2 standard deviations above the mean; and (5) greater than two standard deviations above the mean. Finally, additional performance variables were created to denote whether students passed their NAPLEX and MPJE on the first attempt, achieved a GPA6 below 3.0 on a 4-point scale, and failed to graduate on time with their entering class. For students who took longer than six semesters to complete the program, the GPA data used was their cumulative GPA at the completion of all didactic instruction.

A correlation matrix was created for the primary performance variables. Guidance provided by the British Medical Journal was used to determine the strength of the Pearson correlation for this study. The journal contends that "If we wish to label the strength of the association, for absolute values of $\mathrm{r}, 0-0.19$ is regarded as very weak, 0.20.39 as weak, $0.40-0.59$ as moderate, $0.6-0.79$ as strong and $0.8-1$ as very strong correlation. .."12 The purpose of this analysis was to determine whether the ASMA scores were correlated to the proxies for failure to demonstrate competency. Correlations were run using the EZAnalyze add-in for Microsoft Excel.

Systat 13 (Systat Software Inc., San Jose, CA) was used to perform a linear regression analysis of each of the ASMA examinations (P1-P4) to NAPLEX, MPJE, and GPA6. The effect size estimate calculated used was $\mathrm{R}^{2}$. Cohen posited that $\mathrm{R}^{2}$ of $(0.10)$ equated to a small effect, $(0.30)$ equated to a medium effect, and $(0.60)$ equated to a large effect. ${ }^{13} \mathrm{~A}$ linear regression was used to quantify the relationship between the two sets of continuous variables.
A single-factor ANOVA analysis was performed comparing the ASMA examination z-score groups (1-5) to performance on the MPJE, NAPLEX, and GPA6. The Eta-Squared was calculated to estimate effect size. EtaSquared is similar to the $\mathrm{R}^{2}$ calculation and both describe the percentage of the observed effect that can be attributed to membership in the group being analyzed. ${ }^{14}$ This analysis determined whether poor performers (defined as outliers 2 SD below average) on the ASMA examinations were more likely to fail in the future. While this dichotomized data set is not appropriate as an unbiased estimate of the relationship between the continuous variables, such as ASMA score to GPA, it can be used in concert with the regression analysis to further describe the relationship were the regression results both statistically significant, as evidenced by the $p$ value, and practically significant, as evidenced by the effect size. ${ }^{14}$

Finally, SYSTAT13 was used to perform a cross tabulation analysis and compute odds ratios. This analysis determined the 2-way comparison between student performance on the ASMA examination and performance on the selected proxies for failure. The cut score performance variables for each ASMA examination were compared to NAPLEX Failure, MPJE Failure, GPA6 under 3.0, and Failure to Graduate on time. This analysis was done to determine whether students who fail to master the ASMA examination were more likely to fail to demonstrate competence on the proxies for failure. This element becomes the most important evidence of consequential validity.

\section{RESULTS}

The ASMA examinations all correlated positively to GPA6 and NAPLEX score, with a much weaker association to the MPJE. The P4 ASMA examination scores strongly correlated to NAPLEX and GPA6 and weakly correlated to MPJE. The P3, P2, and P1 examinations moderately correlated to NAPLEX and GPA6, but weakly correlated to MPJE. All correlations were significant with $p$ values $<0.01$ (Table 1).

The regression analyses show that all four ASMA examinations (P1-P4) are significant predictors of NAPLEX, GPA, and MPJE (Figures 1, 2, and 3). The effect size estimates for NAPLEX and GPA in general indicated that the practical significance of the regression was larger for these two variables than for MPJE. The ASMA 1 examination showed a small effect size for NAPLEX and GPA while ASMA 2-4 showed medium effect size.

A graphic representation of the ANOVA analysis of each ASMA examination's z-score groups to NAPLEX and GPA6 is shown in Figures 4 and 5. The average poor performing student (-2 SD below average) on the ASMA 
American Journal of Pharmaceutical Education 2016; 80 (8) Article 135.

Table 1. Correlation of ASMA Exam Scores to NAPLEX, MPJE and GPA

\begin{tabular}{|c|c|c|c|c|c|c|}
\hline & P1 ASMA & P2 ASMA & P3 ASMA & P4 ASMA & NAPLEX Scaled Score & MPJE Scaled Score \\
\hline P2 ASMA & .64 & 1 & & & & \\
\hline $\mathrm{N}$ & 285 & & & & & \\
\hline $\mathrm{P}$ & .01 & & & & & \\
\hline P3 ASMA & 61 & .78 & 1 & & & \\
\hline $\mathrm{N}$ & 279 & 279 & & & & \\
\hline $\mathrm{P}$ & .01 & .01 & & & & \\
\hline P4 ASMA & .59 & .68 & .77 & 1 & & \\
\hline $\mathrm{N}$ & 260 & 260 & 259 & & & \\
\hline $\mathrm{P}$ & .01 & .01 & .01 & & & \\
\hline NAPLEX-SS & .46 & .56 &. .60 &. .70 & 1 & \\
\hline $\mathrm{N}$ & 263 & 263 & 262 & 250 & & \\
\hline $\mathrm{P}$ & .01 & .01 & .01 & .01 & & \\
\hline MPJE SS & .13 & .21 & .27 & .30 & .45 & 1 \\
\hline $\mathrm{N}$ & 249 & 249 & 248 & 237 & 248 & \\
\hline $\mathrm{P}$ & .05 & .01 & .01 & .01 & .01 & \\
\hline Cumulative GPA & .53 & .59 & .57 & .64 & .59 & .30 \\
\hline $\mathrm{N}$ & 284 & 283 & 279 & 260 & 263 & 249 \\
\hline $\mathrm{P}$ & .01 & .01 & .01 & .01 & .01 & .01 \\
\hline
\end{tabular}

ASMA $=$ Wingate Annual Skills Mastery Assessment Exam

NAPLEX-SS = North American Pharmacy Licensure Exam Scaled Score

MPJE $=$ Multistate Jurisprudence Exam Scaled Score

Boldface Pearson Correlation Numbers denote strong correlations above 0.6

examinations failed to achieve a GPA6 above 3.0 and scored poorly on the NAPLEX. Results for the MPJE were not significant. The odds ratio analysis showed mixed results. The ASMA examination cut scores show no significant ability to predict MPJE failure, graduation on time, or cumulative didactic GPA below 3.0 with the exception of the P2 examination. Scoring below or within 1 SEM of the cut score on the P2 examination identified students at significantly higher risk for failing to graduate on time (OR 90) or earning a GPA6 below 3.0 (OR 9.69). Failing to master any of the ASMA examinations identified students at significantly higher risk of failure on the NAPLEX examination on their first attempt (Table 2).

\section{DISCUSSION}

While the regression analysis shows strong evidence of a convergent significant relationship between the ASMA examination scores and the NAPLEX, MPJE, and GPA6, the practical significance of these relationships is confirmed by the evidence of consequential validity provided by the odds ratio analysis. Students who score at or near the cut score on the ASMA examinations were much more likely to fail in the future. Each of the ASMA examinations demonstrated a significant ability to predict ability to pass the NAPLEX examination on the student's first attempt. The P2 examination in particular appears to show promise as a critical predictor of failure.
It is noteworthy that this examination seems to discern to a greater degree than either the $\mathrm{P} 3$ or $\mathrm{P} 4$ examination.

The correlation evidence and regression analysis provide even more evidence that the ASMA examinations appear to possess validity for their intended purpose. Similar studies in medical education compare progress-type examinations to measures of competency. Taylor et al analyzed the relationship between USMLE Step 1 and Step 2 examinations and GPA with the performance rating new physicians earned from their residency directors and found the following Pearson correlations: USMLE Step 1 (.30), USMLE Step 2 (.37), and GPA (.46). ${ }^{15}$ Wilkinson et al analyzed the relationship between the International Foundation of Medical Clinical Science exam developed by the National Board of Medical Examiners and the GPA of 426 Australian medical students and found a correlation of $(0.55) .{ }^{16} \mathrm{McC}$ askill et al compared scores on the USMLE step examination to pediatric board certification examinations and found Pearson correlation of $(0.67)$ for the step 1 and (0.62) for the step 2 examinations. ${ }^{17}$ In a pharmacy education example, Meszaros et al compared students' results on the PCOA examination to advanced practice experience grades and found a Pearson correlation of (0.34) with an $\mathrm{R}^{2}$ effect size of (0.11). ${ }^{18}$ None of these studies are identically constructed and comparing $\mathrm{R}^{2}$ between different studies is not appropriate. But the evidence of correlation of the ASMA examinations to NAPLEX and GPA combined with the 
American Journal of Pharmaceutical Education 2016; 80 (8) Article 135.
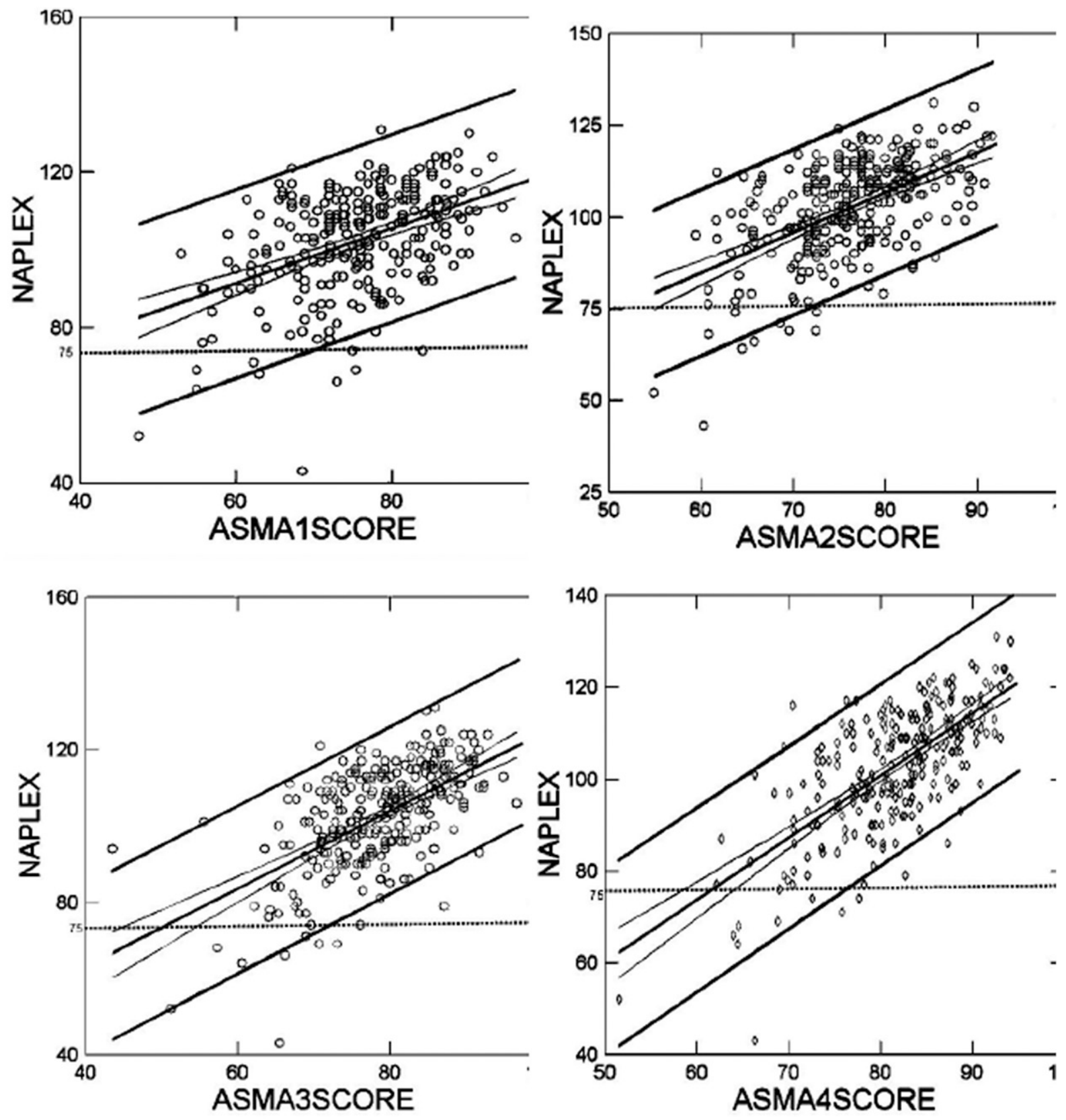

\begin{tabular}{llcl} 
ASMA & No. & $\mathbf{R}^{2}$ & $p$ Value \\
\hline 1 & 263 & 0.21 & $<0.001$ \\
2 & 263 & 0.32 & $<0.001$ \\
3 & 262 & 0.36 & $<0.001$ \\
4 & 250 & 0.48 & $<0.001$ \\
\hline
\end{tabular}

Dark lines represent the Upper and Lower Prediction intervals at $95 \%$ confidence

Grey Straight Lines represents the regression trend line

Grey Curved Lines represent the $95 \%$ confidence intervals

Dotted gray line equals passing score on the NAPLEX of 75

Figure 1. Linear Regression of Wingate Annual Skills Mastery Assessment Exam (ASMA) Scores to North American Pharmacy Licensure Exam Score with 95\% Confidence Intervels. 
American Journal of Pharmaceutical Education 2016; 80 (8) Article 135.
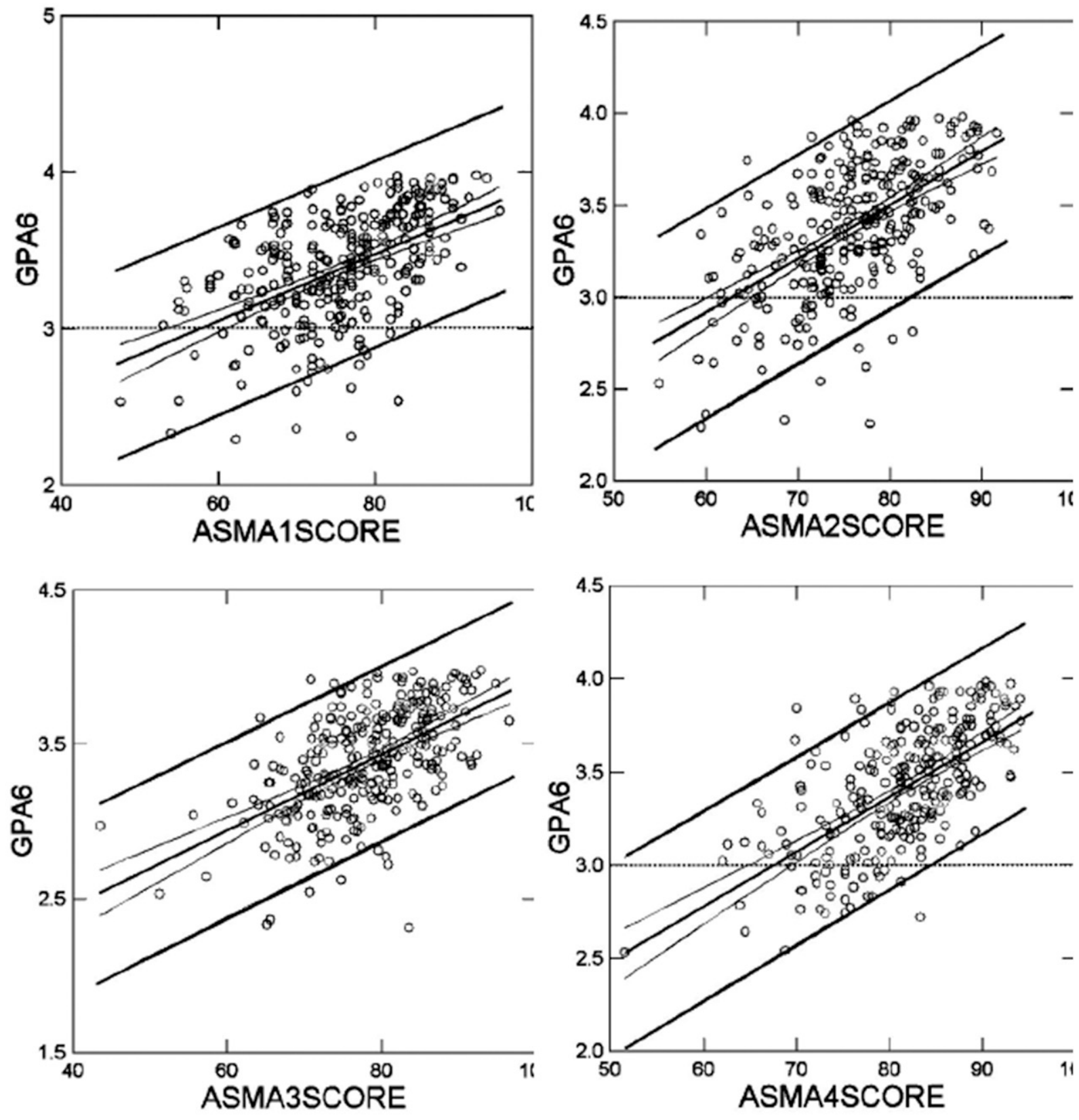

\begin{tabular}{llcl} 
ASMA & No. & $\mathrm{R}^{2}$ & $p$ Value \\
\hline 1 & 284 & 0.28 & $<0.001$ \\
2 & 283 & 0.35 & $<0.001$ \\
3 & 279 & 0.32 & $<0.001$ \\
4 & 260 & 0.41 & $<0.001$ \\
\hline
\end{tabular}

Dark lines represent the Upper and Lower Prediction intervals at $95 \%$ confidence Grey Straight Lines represents the regression trend line

Grey Curved Lines represent the $95 \%$ confidence intervals

Dotted gray line equals GPA of 3.0

Figure 2. Linear Regression of Wingate Annual Skills Mastery Assessment Exam (ASMA) Scores to Cumulative Grade Point Average at Semester 6 with 95\% Confidence Intervals. 
American Journal of Pharmaceutical Education 2016; 80 (8) Article 135.
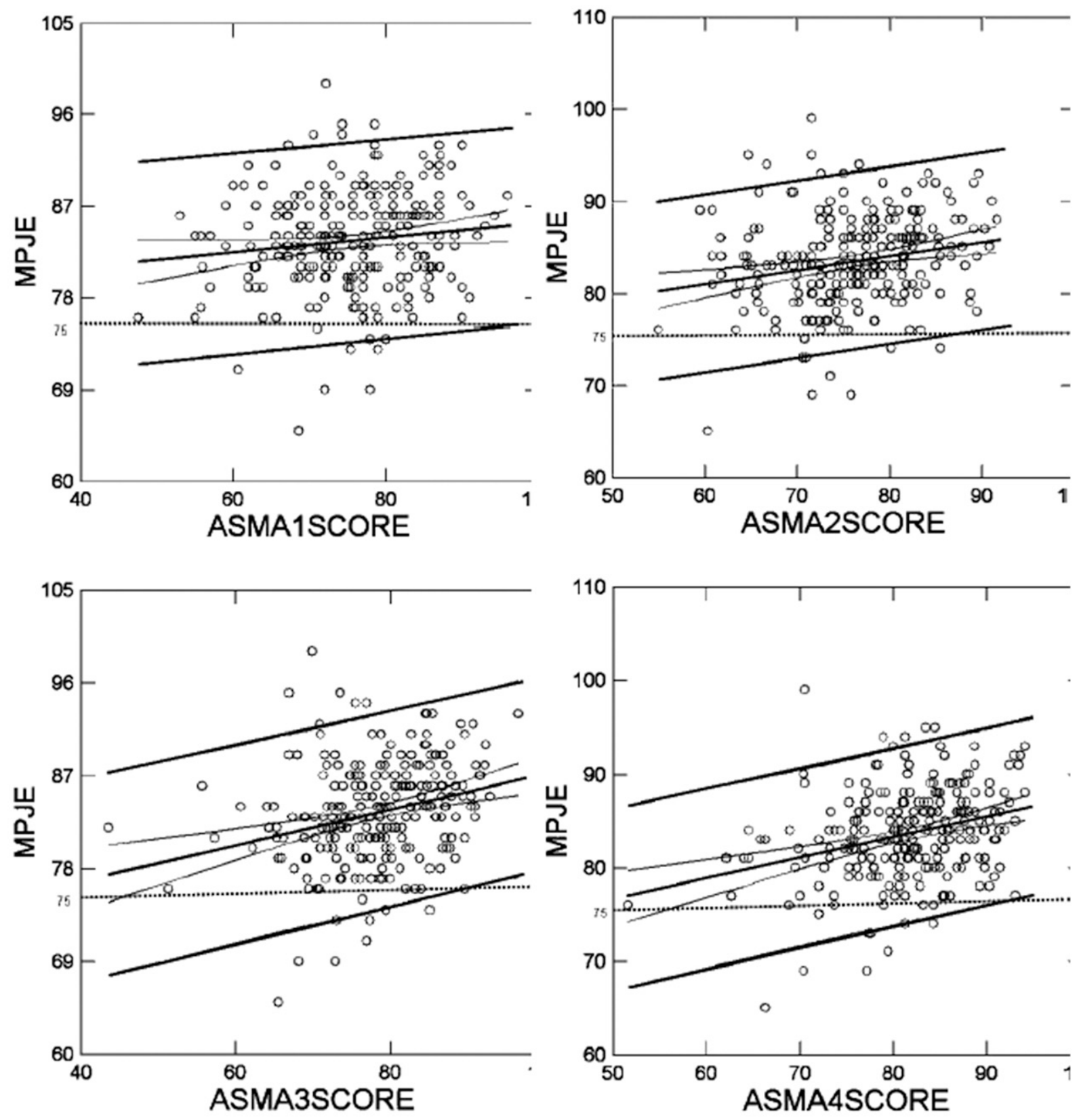

\begin{tabular}{llcc} 
ASMA & No. & $\mathbf{R}^{2}$ & $p$ Value \\
\hline 1 & 249 & 0.016 & 0.045 \\
2 & 249 & 0.045 & 0.001 \\
3 & 248 & 0.073 & 0.001 \\
4 & 237 & 0.092 & 0.001 \\
\hline Dark lines represent the Upper and Lower Prediction intervals at $95 \%$ confidence \\
Grey Straight Lines represents the regression trend line \\
Grey Curved Lines represent the 95\% confidence intervals \\
Dotted gray line equals passing score on the MPJE exam of 75
\end{tabular}

Figure 3. Linear Regression of Wingate Annual Skills Mastery Assessment Exam (ASMA) Scores to Multistate Jurisprudence Exam (MPJE) Scores with 95\% Confidence Intervals. 
American Journal of Pharmaceutical Education 2016; 80 (8) Article 135.
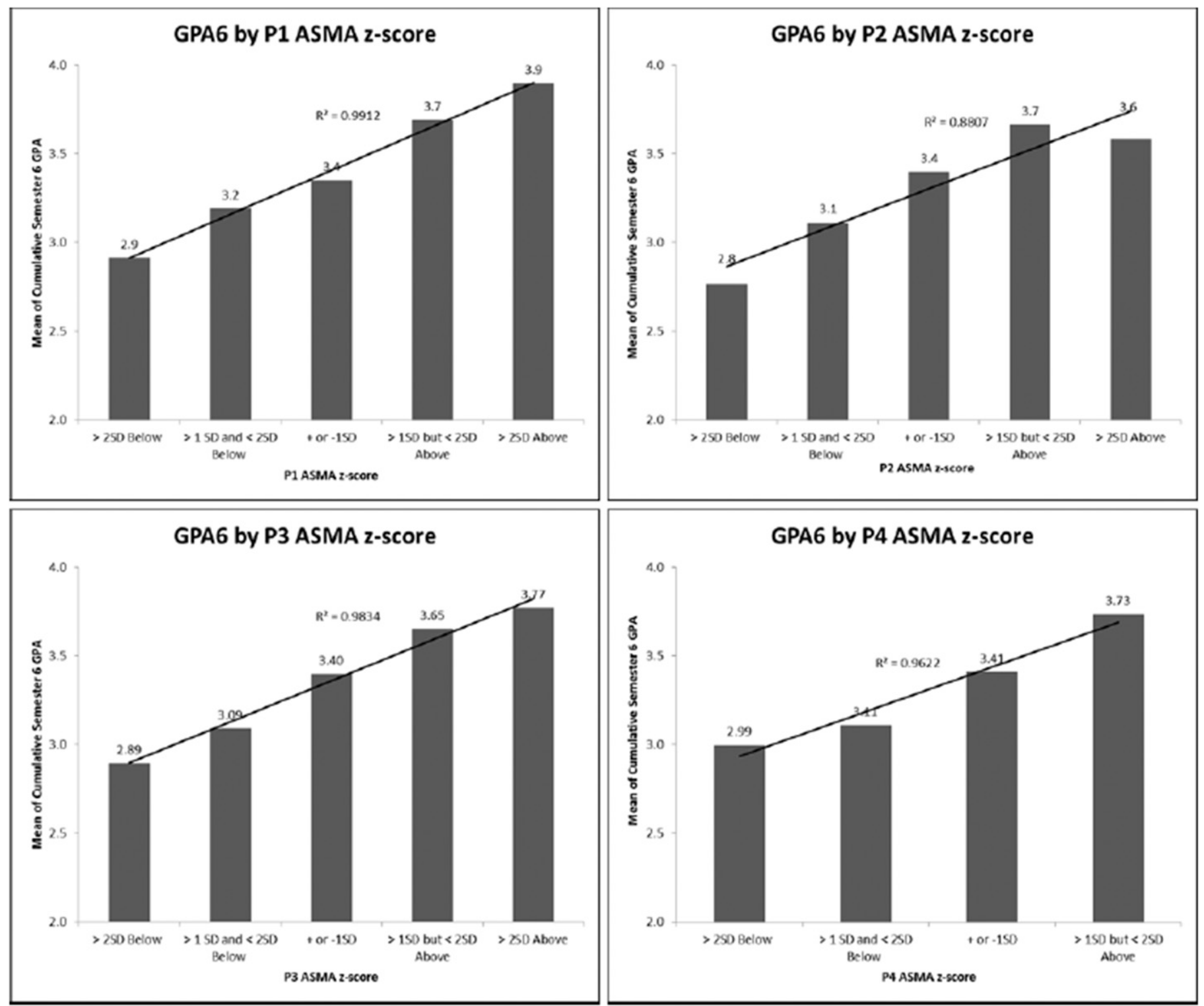

$>2 \mathrm{SD}$ below $=$ at least 2 Standard deviations below the mean, $>1 \mathrm{SD}$ and $<2 \mathrm{SD}=$ more than 1 Standard deviation below the mean but not more than $2,+$ or $-1 \mathrm{SD}=$ between 1 standard deviation above the mean and 1 below the mean, $>1 \mathrm{SD}$ but $<2 \mathrm{SD}=$ more than 1 standard deviation above the mean but not more than 2,>2SD = more than 2 standard deviations above the mean

Figure 4. Wingate Annual Skills Mastery Assessment Exam (ASMA) z-scores to Grade Point Average 6 (GPA6).

medium effect size estimates suggests that the ASMA examinations are at least as useful as other widely studied examinations. For instance, all four ASMA examinations demonstrated both larger Pearson correlations and effect sizes when compared to NAPLEX and Cumulative GPA than the study of PCOA reported by Meszaros. ${ }^{18}$ Thirdyear ASMA correlates to GPA6 (0.57) with $\mathrm{R}^{2}(0.32)$ while PCOA correlates to APPE grades (0.34), with an effect size of (0.11). The correlations to external grading measures were in a similar range with the studies of the USMLE. The P4 ASMA examination correlated to the
NAPLEX (0.70) at least as well as McCaskill's study of the USMLE to pediatric board examination scores (0.67).

The ANOVA analysis as a stand-alone measure of the relationship between the ASMA examinations and the chosen markers for failure would be questionable. MacCallum concluded that the process of dichotomizing continuous variables could lead to negative consequences, including the loss of information regarding individual differences, loss of power, and loss of effect size accuracy. This could lead to overemphasizing the effect size in cases where the grouping results in cohorts with 

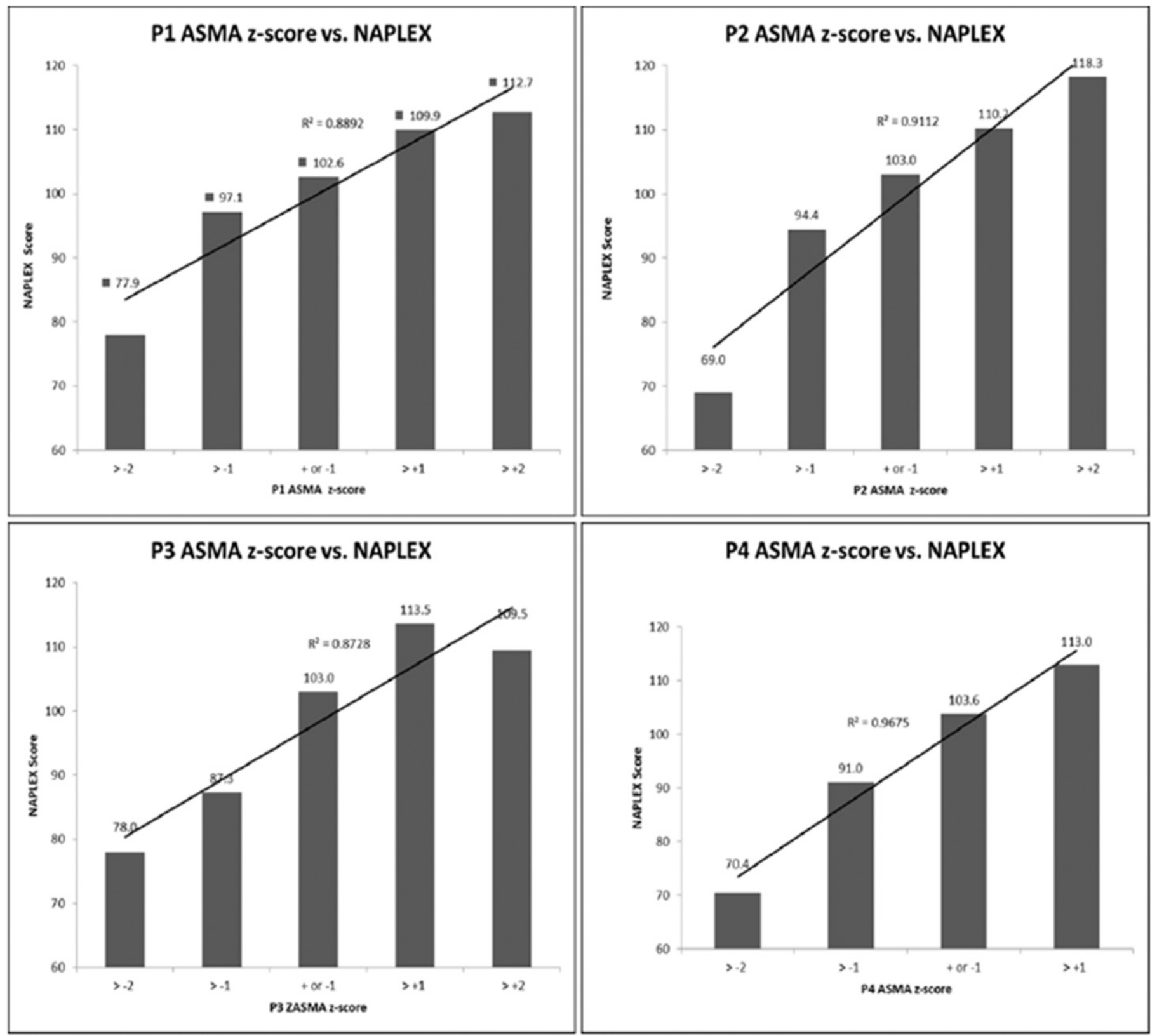

$>-2=$ at least 2 Standard deviations below the mean, $>-1=$ more than 1 Standard deviation below the mean but not more than 2 ,

$+o r-1$ = between 1 standard deviation above the mean and 1 below the mean, $>1=$ more than 1 standard deviation above the mean but not more than $2,>2=$ more than 2 standard deviations above the mean

Figure 5. Wingate Annual Skills Mastery Assessment Exam (ASMA)z-scores vs North American Pharmacy Licensure Exam (NAPLEX).

insufficient sample size. ${ }^{19}$ In this study, the relationship between the continuous variables was first confirmed with a linear regression of the entire data set with $\mathrm{N}$ ranging from 237 to 284 , so the ANOVA should be considered in context with the regression findings. By breaking the continuous data for student performance on the ASMA examinations into z-scores, this ANOVA analysis helped to identify whether students who performed poorly on the ASMA examinations also performed poorly on the selected proxies for failure. Figures 4 and 5 identify a trend that students who performed poorly on the ASMA examinations also performed poorly on NAPLEX and GPA6. While the trend also existed for the MPJE, the low performing cohort did not fail on average, making the finding less significant.

The surprise from the ANOVA analysis was the ability of the P1 and P2 ASMA examinations to identify the group of performers who failed to pass the NAPLEX. The P1 examination identified a cohort of nine students who averaged 77.9 on the NAPLEX, eight of whom failed, and 


\section{American Journal of Pharmaceutical Education 2016; 80 (8) Article 135.}

Table 2. Odds Ratio Analysis of ASMA Exam Mastery vs NAPLEX Failure

\begin{tabular}{|c|c|c|}
\hline ASMA Exam Score & Odds & CI \\
\hline \multicolumn{3}{|l|}{ ASMA 4 Mastered } \\
\hline ASMA4 + 1 SEM $^{1}$ & 96.8 & $2.7-6.5$ \\
\hline ASMA4 + 2 SEM $^{1}$ & 80 & $2.8-6.0$ \\
\hline ASMA3 Mastered & 31.75 & $1.4-5.5$ \\
\hline ASMA $3+1$ SEM $^{1}$ & 27 & $1.6-5.0$ \\
\hline ASMA3 + 2 SEM $^{1}$ & 16.07 & $1.4-4.1$ \\
\hline \multicolumn{3}{|l|}{ ASMA2 Mastered } \\
\hline ASMA2 + 1 SEM $^{1}$ & 15.3 & $1.2-4.3$ \\
\hline ASMA $2+2$ SEM $^{1}$ & 16 & $1.4-4.1$ \\
\hline ASMA1 Mastered & 17.8 & $1.3-4.5$ \\
\hline \multicolumn{3}{|l|}{ ASMA1 + 1 SEM $^{1}$} \\
\hline ASMA1 + 2 SEM $^{1}$ & & \\
\hline
\end{tabular}

ASMA $=$ Annual Skills Mastery Assessment Exam

$\mathrm{SEM}^{1}=$ Standard Error of Measurement

"Mastered"=Above cut score

$\mathrm{CI}=$ Confidence Intervals

Nonsignificant findings left blank

the P2 examination identified a cohort of six students who averaged a 69 on the NAPLEX. They all failed their first attempt. This confirms the ASMA examinations are strong indicators of failure to demonstrate competency by passing the NAPLEX on the first attempt. However, the results are potentially biased by the small sample size of students who failed.

The school can predict which students are 18 times more likely to fail their NAPLEX by reviewing ASMA examination results at the end of their P1 year to determine students who failed to master the examination. This cohort of students varies from two to six individuals in any given year. These students must meet with their advisor and the administration to craft a remediation plan. Identifying these students is relatively easy. Crafting a customized and effective strategy that works for any one individual student has proven significantly more challenging and will require additional study.

Earlier articles established the validity of the process used to construct the ASMA examination to be certain that it was connected to competency-based outcomes and validated the reliability of the cut score development process. ${ }^{6,7}$ The data in this analysis provides compelling evidence that the ASMA examinations assess what they purport to assess, which is a reasonable estimate of the minimal level of competency of pharmacy students at WUSOP.

While the data presented here for WUSOP is not generalizable to any other school, the methodology is. No one assessment tool is capable of providing all of the assessment data a program needs to provide excellent education, and the ASMA examination is no exception. Standards 2016 make the administration of the PCOA examination mandatory for students who have completed their didactic curriculum. In Appendix 3 of the ACPE Standards, the PCOA is solely designed to formatively assess Standard 1-the knowledge of the essential content listed in Appendix 1.2

Administering the PCOA does not, therefore, absolve a program of the responsibility to formatively and summatively assess Standards 2, 3, and 4. While no multiple-choice examination is likely capable of assessing all four standards, the ASMA examination thoroughly engages Standard 2 and the problem-solving elements of Standard 3. In addition, the ASMA examination provides WUSOP with accurate, actionable data to identify students likely to fail, while simultaneously providing the curriculum committee with validation for the achievement of learning outcomes in Standards 1, 2, and elements of 3 . This ability to predict failure provides indirect evidence of student performance on Standards 3 and 4, as many of the students who fail to complete the program have exhibited nonacademic deficiencies in such areas as communications and professionalism.

All student competence studies are hampered by the inability to assess competence as a working professional. Therefore, pharmacy schools are left with NAPLEX scores as a proxy that may or may not truly assess competence. While the sample size of this study is reasonable $(\mathrm{N}=250)$, it still represents only four graduating classes. Because the number of failures is so small (less than $10 \%$ of the population), there is potential for significant measurement error. The ASMA examinations are subject to potential measurement error and the internal reliability of the examinations has room for significant improvement. The school lacks the resources and budget to build an examination with the rigor and psychometric stability of the NAPLEX or PCOA; however, this locally developed examination has proven to provide significant and useful data to improve the quality of the WUSOP program. The ASMA examination program is not universally employed, so it represents only one school's result. However, given the level of scholarship developed to validate the ASMA program, there is strong evidence that this type of progress examination should be strongly considered as a valid assessment of student failure to demonstrate competence.

The PCOA is now a mandatory component in every pharmacy program's assessment toolbox and must be administered to all students in their final semester of didactic education. It assesses only a selected subset of the curriculum, primarily Center for the Advancement of Pharmacy Education (CAPE) 2013 Domain 1, and provides no competency benchmark. The PCOA is norm-referenced and as such allows a student and a program to compare themselves to previous test takers on the foundational knowledge 


\section{American Journal of Pharmaceutical Education 2016; 80 (8) Article 135.}

required to pursue advanced practice experiences. ${ }^{7}$ As a criterion referenced examination tailored to the specific curriculum of the WUSOP program and benchmarked to a faculty-determined minimal competency score, the ASMA examinations attempt to assess whether or not a student is competent. Regardless of use of PCOA, programs will still need to develop evidence of student competence. The ASMA should be considered, along with objective structure clinical examinations and other assessments as ways a program can provide evidence of student competence. Standards 2016 requires the PCOA to be administered in the last semester of every student's didactic education. The results of this analysis suggest that the best time for administration may be earlier in the program. The P2 ASMA examination strongly identified candidates likely to fail and leaves sufficient time before the end of didactic instruction for remediation to be successful.

\section{CONCLUSIONS}

The Wingate University School of Pharmacy Annual Skills Mastery Assessment (ASMA) Exam is given to all pharmacy students each year and is built around gradespecific content and constructs that demonstrate professional competence. Scores from these examinations were strongly correlated to GPA and national licensing examination scores and weakly correlated to law examination scores. Regression analysis indicated a significant linear relationship between examination scores and both GPA and the licensing examinations. Students who performed poorly on the progress exams were more likely to fail the national licensing examination, more likely to fail to graduate on time, and more likely to earn a cumulative didactic GPA below 3.0. The P2 examination program strongly predicts students at risk for failure to graduate on time or achieve a GPA below 3.0, while all four examinations (P1-P4) identify students at risk of failing the national licensing examination on their first attempt. Thus, there is strong evidence that the ASMA examination program is a valid assessment of failure to demonstrate competency on the part of entry level PharmD graduates.

\section{REFERENCES}

1. Vyas D, Halilovic J, Kim MK, Ravnan MC, Rogan EL, Galal SM. Use of cumulative assessments in US schools and colleges of pharmacy. Pharmacy. 2015;3(2):27-38.
2. Accreditation Council for Pharmacy Education. Accreditation standards and key elements for the professional program in pharmacy leading to the doctor of pharmacy degree. https://www.acpe-accredit. org/pdf/Standards2016FINAL.pdf. Accessed 14 July 2015.

3. Downing SM. Validity: on the meaningful interpretation of assessment data. Med Educ. 2003;37(9):830-837.

4. American Educational Research Association, et al. Background. In: Standards for Educational and Pscychological Testing. Washington, DC: American Educational Research Association; 2014:11.

5. Plaza CM. Progress examinations in pharmacy education. Am J Pharm Educ. 2007;71(4):Article 66.

6. Alston GA, Love BL. Development of a reliable, valid annual skills mastery assessment examination. Am J Pharm Educ. 2010;74 (5):Article 80.

7. Alston GA, Haltom WR. Reliability of a minimal competency score for an annual skills mastery assessment. Am J Pharm Educ. 2013;77(10):Article 211.

8. Tavakol M, Dennick R. Making sense of Cronbach's alpha. Int J Med Educ. 2011;2:53-55.

9. Harvill LM. Standard error of measurement. Educational Measurement: Issues \& Practice. 1991;10(2):33-41.

10. Chisholm-Burns MA, Spivey CA, McDonough S, Phelps S, Byrd D. Evaluation of student factors associated with Pre-NAPLEX scores. Am J Pharm Educ. 2014;78(10):Article 181.

11. Hill LH, Delafuente JC, Sicat BL, Kirkwood CK.

Development of a competency-based assessment process for Advanced Pharmacy Practice Experiences. Am J Pharm Educ. 2006;70(1):Article 1.

12. Correlation and Regression. BMJ. http://www.bmj.com/about-bmj/ resources-readers/publications/statistics-square-one/11-correlationand-regression. Accessed June 23, 2015.

13. Cohen J. A power primer. Psychol Bull. 1992;112(1):155-159.

14. Lakens D. Calculating and reporting effect sizes to facilitate cumulative science: a practical primer for t-tests and ANOVAs. Front Psychol. 2013;4:1-12.

15. Taylor ML, Blue AV, Mainous AG III, Geesey ME, Basco WT Jr. The relationship between the National Board of Medical

Examiners' prototype of the Step 2 clinical skills exam and interns' performance. Acad Med. 2005;80(5):496-501.

16. Wilkinson D, Schafer J, Hewett D, Eley D, Swanson D. Global benchmarking of medical student learning outcomes?

Implementation and pilot results of the International Foundations of Medicine Clinical Sciences Exam at The University of Queensland, Australia. Med Teach. 2014;36(1):62-67.

17. McCaskill QE, Kirk JJ, Barata DM, Wludyka PS, Zenni EA, Chiu TT. USMLE Step 1 scores as a significant predictor of future board passage in pediatrics. Ambul Pediatr. 2007;7(2):192-195.

18. Meszaros K, Barnett MJ, McDonald K, et al. Progress examination for assessing students' readiness for Advanced Pharmacy Practice Experiences. Am J Pharm Educ. 2009;73(6): Article 109.

19. MacCallum RC, Zhang S, Preacher KJ, Rucker DD. On the practice of dichotomization of quantitative variables. Psychol Methods. 2002;7(1):19-40. 
American Journal of Pharmaceutical Education 2016; 80 (8) Article 135.

Appendix 1. Individual Mastery Report for Sample Individual Student Report

\begin{tabular}{|c|c|c|c|c|c|}
\hline \multicolumn{6}{|c|}{ Test: P4 2014 Final 2.0; Grade: M; Score: 78.4\% (156.0 of 199.0) } \\
\hline \multicolumn{6}{|c|}{ Terminal abilities acquired by the end of $\mathrm{P} 1$ year } \\
\hline Ability 26 Drug Mechanisms & ;:) & 15.0 of 15.0 & 100.0 & & | \\
\hline Ability 28 Biomedical Informatics & ;:) & 9.0 of 10.0 & 90.0 & & \\
\hline Ability 33 Patient Counseling & ;) & 8.0 of 10.0 & 80.0 & & \\
\hline Ability 60 Drug Pharmacokinetics & ;) & 14.0 of 15.0 & 93.3 & & | \\
\hline Ability 62 Dosing Adjustment & ;) & 7.0 of 9.0 & 77.8 & & \\
\hline Ability 66 Patient Care Plan & ;:) & 9.0 of 10.0 & 90.0 & & | \\
\hline Ability 71 Clinical Labs & :: & 4.0 of 10.0 & 40.0 & & \\
\hline Ability 87 Patient Therapy & 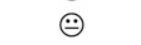 & 6.0 of 10.0 & 60.0 & & \\
\hline P3 Abilities & ;:) & 32.0 of 38.0 & 84.2 & & \\
\hline P4 Abilities & ;:) & 43.0 of 62.0 & 69.4 & & \\
\hline \multicolumn{6}{|c|}{ Terminal abilities acquired by the end of $\mathrm{P} 4$ year } \\
\hline Ability 176 Patient Problem & ;) & 17.0 of 22.0 & 77.3 & & \\
\hline Ability 178 Adverse Drug Effects & $-:$ & 12.0 of 20.0 & 60.0 & & \\
\hline Ability 180 Dosage Adjustment & ;) & 6.0 of 10.0 & 60.0 & & \\
\hline Ability 184 Disease Differential & ;) & 8.0 of 10.0 & 80.0 & & \\
\hline
\end{tabular}

${ }^{a}$ Smiling faces indicate Mastery. Half frowning faces indicate Partial Mastery. Frowning faces indicate No Mastery

${ }^{b}$ Vertical black lines are visual representations of student's scores for each ability. Black bars are visual representations of minimal competency. A vertical bar to the right of a black bar indicates the student score indicates mastery 
American Journal of Pharmaceutical Education 2016; 80 (8) Article 135.

Appendix 2. Mastery Summary Report: P4 2014 Final 2.0 from Scores Spring 2014

\begin{tabular}{|c|c|c|c|c|c|c|c|c|c|c|}
\hline Objective & \#M & \#PM & \#NM & $\% \mathbf{M}$ & $\% \mathbf{P M}$ & $\% \mathrm{NM}$ & PTS & HIGH & LOW & AVG \\
\hline P1 Abilities & 68 & 0 & 0 & 100.0 & 0.0 & 0.0 & 45.0 & 44.0 & 29.0 & 39.2 \\
\hline Ability 9 Pharmacy Calculations & 68 & 0 & 0 & 100.0 & 0.0 & 0.0 & 10.0 & 10.0 & 7.0 & 9.1 \\
\hline Ability 26 Drug Mechanisms & 59 & 5 & 4 & 86.8 & 7.4 & 5.9 & 15.0 & 15.0 & 6.0 & 11.8 \\
\hline Ability 28 Biomedical Informatics & 66 & 2 & 0 & 97.1 & 2.9 & 0.0 & 10.0 & 10.0 & 6.0 & 9.1 \\
\hline Ability 33 Patient Counseling & 67 & 1 & 0 & 98.5 & 1.5 & 0.0 & 10.0 & 10.0 & 6.0 & 9.3 \\
\hline P2 Abilities & 68 & 0 & 0 & 100.0 & 0.0 & 0.0 & 54.0 & 51.0 & 31.0 & 43.5 \\
\hline Ability 60 Drug Pharmacokinetics & 63 & 2 & 3 & 92.7 & 2.9 & 4.4 & 15.0 & 15.0 & 6.0 & 12.3 \\
\hline Ability 62 Dosing Adjustment & 63 & 0 & 5 & 92.7 & 0.0 & 7.4 & 9.0 & 10.0 & 3.0 & 7.4 \\
\hline Ability 66 Patient Care Plan & 67 & 0 & 1 & 98.5 & 0.0 & 1.5 & 10.0 & 10.0 & 4.0 & 8.4 \\
\hline Ability 71 Clinical Labs & 47 & 17 & 4 & 69.1 & 25.0 & 5.9 & 10.0 & 10.0 & 3.0 & 7.2 \\
\hline Ability 87 Patient Therapy & 64 & 4 & 0 & 94.1 & 5.9 & 0.0 & 10.0 & 10.0 & 5.0 & 8.1 \\
\hline P3 Abilities & 68 & 0 & 0 & 100.0 & 0.0 & 0.0 & 38.0 & 38.0 & 25.0 & 33.4 \\
\hline Ability 147 Problem List & 67 & 1 & 0 & 98.5 & 1.5 & 0.0 & 10.0 & 10.0 & 6.0 & 9.3 \\
\hline Ability 148 Patient Care & 67 & 1 & 0 & 98.5 & 1.5 & 0.0 & 15.0 & 15.0 & 8.0 & 13.2 \\
\hline Ability 167 Therapeutic Outcomes & 66 & 2 & 0 & 97.1 & 2.9 & 0.0 & 13.0 & 13.0 & 8.0 & 10.9 \\
\hline P4 Abilities & 65 & 0 & 3 & 95.6 & 0.0 & 4.4 & 62.0 & 58.0 & 37.0 & 48.8 \\
\hline Ability 176 Patient Problem & 64 & 4 & 0 & 94.1 & 5.9 & 0.0 & 22.0 & 22.0 & 13.0 & 17.0 \\
\hline Ability 178 Adverse Drug Effects & 51 & 11 & 6 & 75.0 & 16.2 & 8.8 & 20.0 & 18.0 & 10.0 & 14.8 \\
\hline Ability 180 Dosage Adjustment & 68 & 0 & 0 & 100.0 & 0.0 & 0.0 & 10.0 & 10.0 & 6.0 & 8.0 \\
\hline Ability 184 Disease Differential & 66 & 1 & 1 & 97.1 & 1.5 & 1.5 & 10.0 & 10.0 & 5.0 & 8.9 \\
\hline \multicolumn{11}{|l|}{ Summary } \\
\hline All Objectives & 29 & - & 0 & 42.7 & - & 0.0 & 199.0 & 185.0 & 129.0 & 164.8 \\
\hline All Areas & 65 & - & 0 & 95.6 & - & 0.0 & 199.0 & 185.0 & 129.0 & 164.8 \\
\hline
\end{tabular}

$\# \mathrm{M}=$ Number Mastering; \#PM=Number Partial Mastering; \#NM=Not Mastering; \%M=Percent Mastering; \%PM=Percent Partial Mastering; $\% \mathrm{NM}=$ Percent Not Mastering. PTS=Possible Points; High=Highest Points; Low=Lowest Points; Avg=Average Points 
American Journal of Pharmaceutical Education 2016; 80 (8) Article 135.

Appendix 3. Annual Skills Mastery Assessment Exam Test Blueprint for 2014

\begin{tabular}{|c|c|c|c|c|c|}
\hline Ability Set & Ability & P1 Test & P2 Test & P3 Test & P4 Test \\
\hline \multicolumn{6}{|l|}{ P1 Abilities } \\
\hline \multicolumn{6}{|l|}{ TABO \# } \\
\hline 9 & Perform a selected pharmaceutical calculation & 12 & 10 & 10 & 10 \\
\hline 22 & $\begin{array}{l}\text { Describe the various cellular processes essential for } \\
\text { survival }\end{array}$ & 12 & 0 & 0 & 0 \\
\hline 23 & $\begin{array}{l}\text { Define the structure, nature, location, and function of } \\
\text { a selected component of the human anatomy }\end{array}$ & 12 & 0 & 0 & 0 \\
\hline 25 & $\begin{array}{l}\text { Describe how the organs and organ systems function } \\
\text { together to maintain various homeostatic variables }\end{array}$ & 12 & 10 & 10 & 0 \\
\hline 26 & $\begin{array}{l}\text { Describe the mechanism of action for a selected drug } \\
\text { or drug class }\end{array}$ & 25 & 10 & 10 & 10 \\
\hline 28 & $\begin{array}{l}\text { Describe the appropriate use of primary, secondary, } \\
\text { and tertiary medical literature, databases and } \\
\text { Internet sites }\end{array}$ & 12 & 10 & 10 & 10 \\
\hline 33 & Communicate selected counseling points & 15 & 10 & 10 & 10 \\
\hline \multicolumn{6}{|l|}{ P2 Abilities } \\
\hline \multicolumn{6}{|l|}{ TABO \# } \\
\hline 60 & $\begin{array}{l}\text { Describe the fundamental elements and major factors } \\
\text { that influence drug absorption, distribution, } \\
\text { binding, metabolism, and excretion }\end{array}$ & 0 & 10 & 10 & 10 \\
\hline 62 & $\begin{array}{l}\text { Describe the influence of drug and patient } \\
\text { characteristics on dosing }\end{array}$ & 0 & 10 & 10 & 10 \\
\hline 66 & $\begin{array}{l}\text { Create a plan to appropriately monitor/select drugs } \\
\text { using drug concentrations and physiological effects }\end{array}$ & 0 & 10 & 10 & 10 \\
\hline 71 & $\begin{array}{l}\text { Recognize the clinical significance and implications } \\
\text { of a given laboratory test result }\end{array}$ & 0 & 10 & 10 & 10 \\
\hline 87 & Define a therapeutic goal for a selected patient & 0 & 10 & 10 & 10 \\
\hline 96 & $\begin{array}{l}\text { Detect the adverse drug effects associated with } \\
\text { a selected drug regimen }\end{array}$ & 0 & 10 & 10 & 0 \\
\hline 115 & $\begin{array}{l}\text { Describe the major regulations that influence the } \\
\text { practice of pharmacy in the state of North Carolina }\end{array}$ & 0 & 10 & 10 & 10 \\
\hline \multicolumn{6}{|c|}{ P } \\
\hline \multicolumn{6}{|l|}{ TABO \# } \\
\hline 147 & Create a problem list for a selected patient & 0 & 0 & 10 & 10 \\
\hline 148 & $\begin{array}{l}\text { Design a patient-specific care plan for a selected } \\
\text { patient }\end{array}$ & 0 & 0 & 15 & 15 \\
\hline & Assess therapeutic outcome in a selected patient & 0 & 0 & 15 & 15 \\
\hline \multicolumn{2}{|l|}{ TABO \# } & \multicolumn{4}{|c|}{ P4 Abilities } \\
\hline 175 & $\begin{array}{l}\text { Define a drug and nondrug regimen for the resolution } \\
\text { of the problem identified for a selected patient }\end{array}$ & 0 & 0 & 0 & 10 \\
\hline 176 & $\begin{array}{l}\text { Define an alternative course for the resolution of the } \\
\text { problem identified for a selected patient }\end{array}$ & 0 & 0 & 0 & 15 \\
\hline 178 & $\begin{array}{l}\text { Detect adverse drug effects associated with a selected } \\
\text { drug regimen }\end{array}$ & 0 & 0 & 0 & 15 \\
\hline 180 & $\begin{array}{l}\text { Recommend dosage adjustment for a selected drug } \\
\text { regimen for a selected patient }\end{array}$ & 0 & 0 & 0 & 10 \\
\hline 184 & $\begin{array}{l}\text { Differentiate disease states based upon given } \\
\text { symptoms in a selected patient }\end{array}$ & 0 & 0 & 0 & 10 \\
\hline Item Count & & 100 & 120 & 160 & 200 \\
\hline
\end{tabular}

$\mathrm{TABO}=$ Terminal Ability Based Outcomes Statement selected from the Wingate University School of Pharmacy List of TABO 